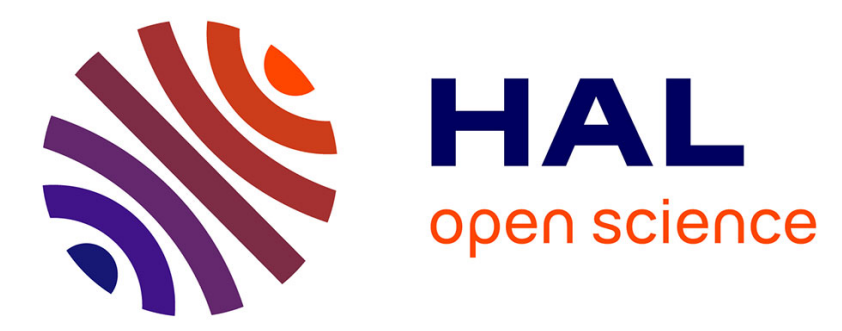

\title{
Local structure of condensed Zinc Oxide
}

F. Decremps, F. Datchi, A. M Saitta, J. Itié, A. Polian, F. Baudelet, S. Pascarelli, A Di Cicco

\section{To cite this version:}

F. Decremps, F. Datchi, A. M Saitta, J. Itié, A. Polian, et al.. Local structure of condensed Zinc Oxide. Physical Review B: Condensed Matter and Materials Physics (1998-2015), 2003, 68 (10), 10.1103/PhysRevB.68.104101 . hal-01921250

\section{HAL Id: hal-01921250 https://hal.sorbonne-universite.fr/hal-01921250}

Submitted on 13 Nov 2018

HAL is a multi-disciplinary open access archive for the deposit and dissemination of scientific research documents, whether they are published or not. The documents may come from teaching and research institutions in France or abroad, or from public or private research centers.
L'archive ouverte pluridisciplinaire HAL, est destinée au dépôt et à la diffusion de documents scientifiques de niveau recherche, publiés ou non, émanant des établissements d'enseignement et de recherche français ou étrangers, des laboratoires publics ou privés. 


\title{
Local structure of condensed Zinc Oxide
}

\author{
F. Decremps, F. Datchi, A. M. Saitta, J. P. Itié, A. Polian and F. Baudelet \\ Laboratoire de Physique des Milieux Condensés, CNRS-UMR 7602, \\ Université Pierre \& Marie Curie, Tour 13, B7r7, \\ 4, place Jussieu, 75252 Paris CEDEX 05, France
}

S. Pascarelli

E.S.R.F., BP 220, 38043 Grenoble, France

\author{
A. Di Cicco \\ INFM, Dipartimento di Matematica e Fisica, Università di Camerino, \\ via Madonna delle Carceri, 62032 Camerino (MC), Italy
}

(Dated: December 20, 2002)

\begin{abstract}
The high pressure local structure of zinc oxide has been studied at room temperature using combined energy dispersive X-ray diffraction and X-ray absorption spectroscopy experiments. The structural parameter $u$ and the lattice parameters ratio $\frac{c}{a}$ of the wurtzite phase is given as function of pressure and compared with results from ab-initio calculations based on a plane wave pseudopotential method within the density functional theory. In good agreement with present calculations, our experimental data do not show any variation of $u(\mathrm{P})$ in the low-pressure wurtzite phase between 0 and $9 \mathrm{GPa}$, pressure at which the phase transition to the rocksalt phase occurs. In view of these results, theoretical models identifying the wurtzite-to-rocksalt transition as an homogeneous path are discussed.
\end{abstract}

PACS numbers:

\section{INTRODUCTION}

Aside from being a good sunblock to protect retired scientists from UV overexposure, $\mathrm{ZnO}$ is a transparent piezoelectric semiconductor which occurs naturally as a mineral (called zincite), e.g at the meeting point of three relevant groups for Physics, Materials Science and Geophysics. More specifically, high pressure experimental data on this compound are fundamental to condensedmatter physics because of the strict requirements they place on empirical models of interatomic potential repulsive part in semiconductors, which might afterwards benefit the design of new optoelectronic devices.

As pressure is raised from ambient condition, $\mathrm{ZnO}$ and group-III nitrides (AlN, GaN and $\mathrm{InN}$ ) transform from fourfold (wurtzite, labelled $w-, \mathrm{P}_{3} m c$ space group) to sixfold (rocksalt, labelled $r-, F m \overline{3} m$ space group) coordinated crystal structures. The microscopic mechanism involved in this transformation has been the subject of numerous studies and the existence of a transition path is still debated. Recent theoretical calculations of phonon dispersion have shown that standard total-energy calculations might lead to wrong conclusions on the occurrence of high-pressure structural phase transitions[1, 2]. Moreover, lattice-dynamical properties are helpful in understanding the transformation pathways. For example, $w$-InN was predicted[3] to be unstable prior the transition to the $r$ - high pressure phase, leading to a second order isostructural transformation from the standard wurtzite phase to another hexagonal configuration (labelled $w^{\prime}-$ ). Lately, Serrano et al [4] refined the previous conclusion using DFT-LDA calculations and found a clear variation of the internal crystallographic parameter $u$ from its ambient value $\left(\sim \frac{3}{8}\right)$ up to 0.5 when $w$-AlN and $w$-InN are pressurized. The same type of intermediate phase transformation has been described for $\mathrm{GaN}[5]$ where, via a group theory analysis of the group-subgroup relationship between the two wurtzite networks, a transformation involving coherent atomic motions rather than a simple unpredictable diffusion is proposed. However, this result has not been confirmed neither experimentally nor theoretically [6]. This second-order process has only been calculated for group-III nitrides and the presence of such transformation in $\mathrm{ZnO}$ will be probed and discussed in the present paper (section IV).

The variation of $u$ up to 0.5 has never been observed experimentally, probably because the $w$ - to $w^{\prime}$ - transformation is usually predicted to occur at a pressure above the observed first-order $w$ - to $r$ - transition. Nevertheless, if such a martensitic-like phase transition exists in nitrides or $\mathrm{ZnO}$, it should be preceded by some structural instabilities in the $w$ - phase like a nonlinear pressure behavior of $\frac{c}{a}$ and $u[3]$ or phonons softening [5]. Experimentally, the elastic behavior of $\mathrm{ZnO}$ has been determined by ultrasonic experiments up to the $w$ - to $r$ - transition pressure $(9 \mathrm{GPa})$. An unusual negative value for the shear modes has been observed and indicates that $w$-ZnO is indeed unstable with respect to a shear perturbation [7], result that has been recently confirmed by theory [8]. By contrast, no singularity occurs on any optical mode neither in the experiments nor in the calculations [9]. Thus, an accurate measurement of $u(\mathrm{P})$ in $\mathrm{ZnO}$ would shed some light on the $w$ - structure stability. In addition, the determination of the bond distance $\mathrm{Zn}-\mathrm{O}$ as a function of pressure is the most direct way to explore the repulsive 
part of the interatomic potential, which is of great importance for the understanding of $\mathrm{ZnO}$ physical and chemical properties.

The room temperature compression behavior of $w$ - and $r$-ZnO was studied previously by X-ray powder diffraction [10-15]. However, neither calculation nor experiment on the pressure dependence of the local structure of $w$ - $\mathrm{ZnO}$ has been published. An experimental attempt of $u(\mathrm{P})$ determination has been proposed [11] by comparing the integrated intensity of the (002) and (101) energy-dispersive X-ray diffraction lines. But, according to the author, in view of the problems arising from the extraction of the powder quality (texture, microstrain, orientation) and the pattern background contributions (Compton scattering, fluorescence), more work is needed to obtain reliable data on the local structure. Results from high-pressure ${ }^{67} \mathrm{Zn}$-Mössbauer spectroscopy on $w$ $\mathrm{ZnO}$ allowed to probe the hyperfine interactions related to the valence state of zinc [13]. But, here again, the pressure variation of the microscopic structure can only be deduced indirectly (through the use of lattice dynamics and LAPW band-structure calculations).

The principal objective of this study is to get atomicscale insight into the wurtzite structure stability of condensed $\mathrm{ZnO}$. The pressure variation of the atomic positions in both wurtzite and rocksalt phases are first calculated through the density-functional perturbation theory (DFPT) approach using a standard local-density approximation plane-wave/pseudopotential scheme, and, secondly, probed experimentally by Zn EXAFS. It is well known that EXAFS analysis suffers from the ambiguity that the results are strongly dependent on the number of fitting parameters [16]. This is particularly critical in the case of the wurtzite structure where, at least, contributions up to the fifth shell including multiple-scattering effects have to be taken into account to refine satisfactorily the experimental XAFS spectra. Thus, at the same experimental conditions of pressure, temperature, powder texture and stain-state, X-ray diffraction (XRD) patterns were recorded to follow the long-range order. The present XAS and XRD combined study allows to confirm the reliability of our analysis by checking the pressure dependance of $a(\mathrm{P})$ and $c(\mathrm{P})$ deduced from XAFS[17] against those obtained independently from XRD data treatment.

In addition, high pressure X-ray absorption near edge structure (XANES) measurements on bulk $\mathrm{ZnO}$ were carried out in order to get an accurate determination of the Zn $K$-edge pressure dependence. Up to $\sim 20 \mathrm{GPa}$, no dramatic modification of the $r$-phase edge position indicates the absence of metallization. Further, the structure of both $w$ - and $r$-ZnO under pressure has been confirmed by comparing the XANES raw data with full multiple scattering calculations. In particular, the present study allows to disentangle the ambiguity on the crystallographic structure of the recovered sample at $\mathrm{P}=0$.

Finally, the experimental results are compared with original results from $a b$-initio calculations and discussed in terms of wurtzite stability and microscopic $w$ - to $r$ - transition process.

\section{EXPERIMENTS}

\section{A. Sample and high pressure apparatus}

The high pressure cell was a conventional membrane diamond anvil cell [18]. A steel gasket was preindented from 200 to $60 \mu \mathrm{m}$, and a $150 \mu \mathrm{m}$ hole was drilled in the center by spark-erosion. Polycrystalline zinc oxide with a nominal purity of $99.9995 \%$, purchased from Alfa AESAR was loaded into the gasket hole. Chemically inert, silicon-oil was used as pressure transmitting medium. Pressure was systematically measured in-situ before and after each measurement using the fluorescence emission of a ruby ball $[19,20]$ placed into the gasket hole. The accuracy was better than $0.5 \mathrm{GPa}$ at the maximum pressure reached.

\section{B. X-ray diffraction (XRD)}

X-ray diffraction experiments have been performed at LURE (Orsay, France), where the synchrotron radiation is emitted by the DCI storage ring operating at 1.85 $\mathrm{GeV}$ with typical currents of $300 \mathrm{~mA}$. The powder $\mathrm{x}-$ ray diffraction measurements were made in the energy dispersive mode with the wiggler at DW11 station. After the energy calibration of the $G e$ detector, the $2 \theta$ angle was determined by collecting diffraction patterns of a gold sample placed in between the anvils $\left(2 \theta=13.759^{\circ}\right)$. The polychromatic $\mathrm{x}$-ray beam was collimated to a $50 \times 50$ $\mu \mathrm{m}^{2}$ spot centered on to the gasket hole and the diffraction patterns were collected for up to $600 \mathrm{~s}$.

\section{Extended X-ray absorption fine structure} (EXAFS)

Extended X-ray absorption fine structure spectroscopy has been performed at LURE, at the D11 dispersive XAS station at the $\mathrm{Zn} K$ edge. The calibration of the photon energy scale was determined comparing the $K$ edge pure $\mathrm{Zn}$ spectrum collected at ambient conditions using the dispersive technique with analogous spectra recorded in the same conditions using the standard XAS technique (double-crystal scanning monochromator). The position of the diamond cell was optimized in order to reject strong Bragg peaks from the diamonds at high energy above the absorption edge: the available energy range was up to about $500 \mathrm{eV}$ above the $\mathrm{Zn} K$-edge. More details on the beam-line setup and the procedure of pattern acquisition have been previously published [21]. 


\section{X-ray absorption near edge structure (XANES)}

The high pressure Zn $K$-edge XANES measurements on bulk $\mathrm{ZnO}$ were carried out at the European Synchrotron Radiation Facility on beamline ID24 [22]. A curved $\mathrm{Si}(111)$ crystal polychromator horizontally focused $\mathrm{a} \sim 500 \mathrm{eV}$ fan of radiation onto $\mathrm{a} \sim 30 \mu \mathrm{m}$ spot. A Pt-coated mirror, placed $\sim 60 \mathrm{~cm}$ upstream the sample at a grazing incidence angle of $4 \mathrm{mrad}$, was used to vertically refocus the beam down to below $30 \mu \mathrm{m}$ FWHM [23].

\section{E. Computer experiment: method of calculation}

The high-pressure structural properties of zinc oxide have also been studied by means of $a b$ initio methods. Our calculations have been performed using the PWSCF code[24], and are based on the density-functional theory (DFT) within the local-density approximation[25]. A standard plane-wave/pseudopotential scheme is adopted. Our pseudopotentials are chosen through the TroullierMartins scheme[26]. Other zinc-based semiconductors can accurately be treated by freezing the zinc $d$ electrons in the core, and adopting a non-linear core-correction scheme to account for this approximation. An accurate study of $\mathrm{ZnO}$ requires instead the explicit treatment of the $d$ electrons of $Z n$ as valence electrons. This choice, yet computationally demanding, proves essential in yielding, for example, the correct energetics of the different structures. Our calculations were performed with a kinetic energy cutoff of $75 \mathrm{Ry}$, and special k-points integration in the Brillouin zone, with (444) or (666) regular grids.

The wurtzite structure contains two parameters not fixed by symmetry, i.e. the $c / a$ ratio and the internal coordinate $u$. In order to determine the pressure behavior of the system in the wurtzite phase, we chose to fix different volumes, and to relax the total energy of the crystal with respect to those parameters. This method allows us to obtain an energy-volume equation of state which can be fitted to a Murnaghan equation. The same kind of calculation is straightforwardly performed in the cubic $\mathrm{NaCl}$ phase, where of course the only variable is the lattice parameter.

\section{ANALYSIS}

\section{A. X-Ray Diffraction data}

Figure 1 shows a stack of $w$ - and $r$ - spectra recorded from ambient to high pressure where the background has been extracted. Because the origin of the background is multiple-synchrotron source, detector, absorption of the sample and its environment- and difficult to quantify, it was fitted with a polynomial function up to the third order. Peak positions were determined using a pseudovoigt

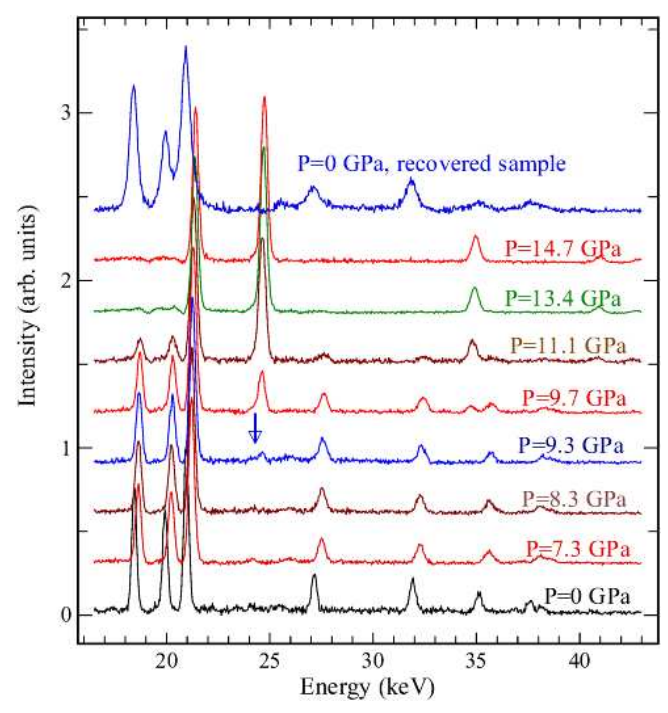

FIG. 1: X-ray energy dispersive spectra for wurtzite and rocksalt $\mathrm{ZnO}$ at different pressure. At $9.3 \mathrm{GPa}$, a new diffraction peak (arrow) reflects the occurence of the $w$ - to $r$ - phase transition. On decompression (from $16 \mathrm{GPa}$ ), the diffraction pattern fingerprint of the $w$ - phase reappears at $\sim 2 \mathrm{GPa}$.

model and the lattice spacings $d_{h k l}$ (in $\stackrel{\circ}{A}$ ) were extracted from the energies of the reflection lines $E_{d h k l}$ (in $k e V$ ) using the Bragg's law: $E_{d h k l} . d_{h k l}=6.199 /(\sin \theta)$. Finally, the lattice parameters were calculated using a classical least-squares refinement program. The relative errors of the lattice parameters were determined to be less than $0.05 \%$ in all refinements.

\section{B. EXAFS}

EXAFS data analysis were carried out in the framework of the GNXAS codes [27-29] for theoretical calculations of x-ray absorption fine structure including multiple-scattering (MS) pathways. The simulations have been performed using the muffin-tin approximation and the Hedin-Lundqvist exchange-correlation selfenergy. The refinement is based on a minimization of a standard $\chi^{2}$-like residual function related to the difference between the raw experimental absorption coefficient and the theoretical signal [30]. Using continued-fraction techniques, the XAFS signal calculations are performed including both two-atom and three-atom irreducible MS terms (respectively noted $\gamma^{(2)}$ and $\gamma^{(3)}$ ) [27]. In the following, using the standard notation, the $n$-body distribution function peaks of the $i^{t h}$ shell will be labelled $g_{i}^{(n)}$. Before fitting the experimental signal with a theoretical model, a pre-analysis has been performed in order to : (1) simulate the atomic background, including the main anomalies due to the opening of double-electron excitation channels; (2) determine for each structure the relevant fitting process. 


\section{Background substraction}

The presence of double-electron photoexcitations (one electron is excited to the continuum state, the other being promoted to the unoccupied discrete orbital) in x-ray absorption spectra is now well established, and has been successfully identified for noble gases, metals in vapor state, solutions or molten elements (see for example appendix A2 of Ref. [29]). For highly ordered samples as zinc oxide, XAFS spectra contain a strong structural signal rendering tricky the extraction of the multi-electron excitation component. However, even tiny, the presence of such features usually results in anomalies in the EXAFS signal background which affect the reliability of the local structure determination.

The study of double-electron excitations in $\mathrm{Zn} K$-edge has only been attempted in the vicinity of the $K$-edge[31]. At highest energy (where the double-electron photoexcitations may create anomalies in the EXAFS signal), the more relevant results have only been obtained on copper[32] and on $4 p$ elements from $Z=31$ (Ga) to $Z=36$ $(\mathrm{Kr})[33]$, where the magnitude of the opening of absorption channels creating the $[1 s 3 p]$ and $[1 s 3 d]$ double-hole configurations is shown to linearly increase with decreasing $Z$. The presence of these channels has also been evidenced for $\mathrm{Ga}$ and $\mathrm{Ge}(Z=32)$, whose contribution appears to be absolutely necessary to get reasonable EXAFS fits [28]. Moreover, the amplitude of the [1s3p] double-electron excitation cross-section has been experimentally shown to be larger when the metal is oxydised (mainly due to the more efficient $1 s$ hole screening in metal than in its corresponding oxide[34]). Thus, the extrapolation of previous results to $Z=30$ ( $\mathrm{Zn}$ ) justifies the background correction procedure used in the present work on $\mathrm{ZnO}$, where the $[1 s 3 p]$ double-electron feature has been taken into account. Experimental EXAFS spectra of $w$-ZnO at ambient conditions and bestfit model background including double-electron features are reported in Figure 2. The empirical background anomaly has been fitted to the experimental data using the "smooth step" function available in the Fitheo program of GNXAS [29]. As empirically expected, the amplitude of the $[1 s 3 p]$ cross-section is significant. Its energy can be roughly estimated using the $Z+1$ rule leading in the case of $\mathrm{Zn}$ to $100 \mathrm{eV}\left(3 p_{3 / 2}\right)$ and $103.5 \mathrm{eV}$ $\left(3 p_{1 / 2}\right)$ above the main 1 s edge. It can be noted that these values are slightly lower than the $[1 s 3 p]$ energy observed in this work $(\sim 9790 \mathrm{eV})$, probably because of a merging between $[1 s 3 s]$ and $[1 s 3 p]$ contributions.

\section{Wurtzite phase analysis}

An initial study revealed by the use of GNXAS code that while a substantial contribution to the EXAFS signal is given by the oxygen nearest neighbors, the additional contributions from the next-nearest neighbors are essential to account for the total EXAFS signal. How-

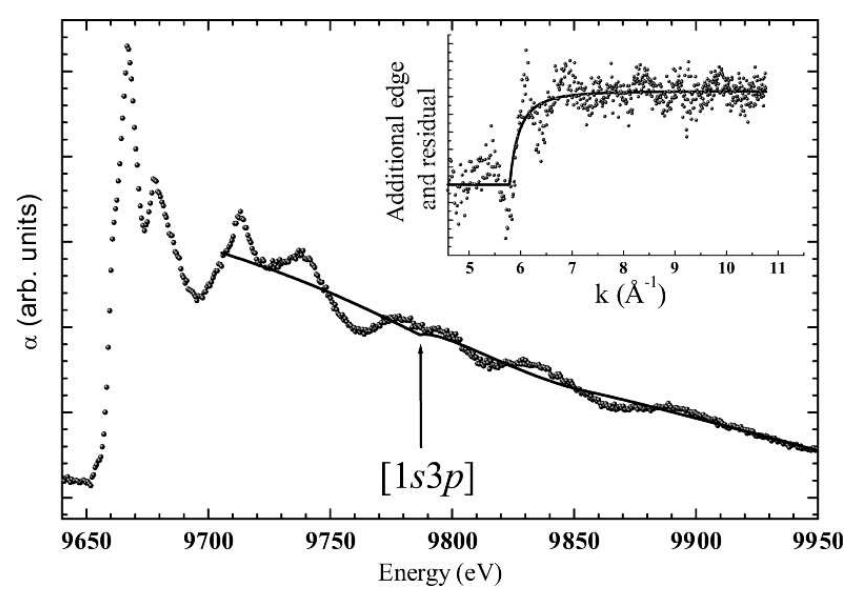

FIG. 2: Raw $K$-edge spectrum of $w$-ZnO at $\mathrm{P}=0$ and background (modelled using a four degree polynomial function) with visible double-electron excitation at $\sim 9790 \mathrm{eV}$. Onset: magnified plot of double-electron excitation shape (line) and residual experimental signal. The background parameters were independently refined for each spectra (different pressure, different structure), and no significant difference was detected. It indicates in particular the expected transferability of double-electron features from a structure $(w-)$ to another $\left(r_{-}\right)$.

ever, increasing the number of fitting parameters dramatically decreases the result accuracy and a strategy has to be developed to choose appropriate fitting parameters and to find connections between them.

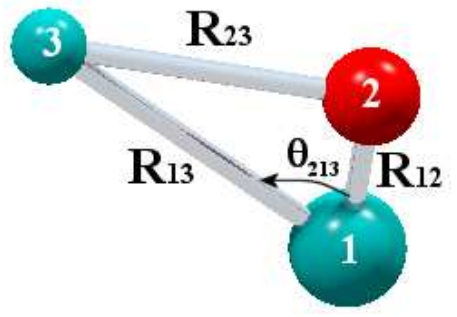

FIG. 3: Schematic representation of a three-body configuration. The corresponding EXAFS signal is specified by the parameters $R_{12}, R_{13}$ and $\theta_{213}$, the third distance $R_{23}$ being calculated through the Carnot formula.

It is obvious that in a general triangular three-atom configuration, the structural parameters $R_{12}, R_{13}$ and $\theta_{213}$ automatically define the third interatomic distance $R_{23}$ (see Figure 3). Therefore, the doublet $\gamma^{(2)}$ signals beyond the second shell can be calculated using the corresponding triplet coordinates. This strategy avoids the use of further structural parameters by introducing in the fitting process an interconnection between the $g^{(2)}$ and the $g^{(3)}$ peaks through the Carnot formula [35]. In the following, the two-atom signals $\gamma^{(2)}$ of the second shell $\left(g_{2}^{(2)}\right.$ peak) and the fourth shell $\left(g_{4}^{(2)}\right.$ peak $)$ have been calculated using the three-atoms contributions $\gamma^{(3)}\left(g_{1}^{(3)}\right.$ 
TABLE I: Pair and triplet configurations for $w$-ZnO at ambient conditions. The three coordinates defining the three-body peaks are as described in Figure 3. The degeneracy (Deg) is specified for each configuration.

\begin{tabular}{|c|c|c|c|c|c|c|}
\hline Peak & $R_{12}(\stackrel{\circ}{A})$ & $R_{13}(\stackrel{\circ}{A})$ & $\theta_{213}\left(^{o}\right)$ & Deg. & Atoms & XAFS signal \\
\hline \multicolumn{7}{|l|}{$g^{(2)}$} \\
\hline 1 & 1.977 & & & 4 & $\mathrm{Zn}-\mathrm{O}$ & $\gamma_{1}^{(2)}$ \\
\hline 2 & 3.207 & & & 6 & $\mathrm{Zn}-\mathrm{Zn}$ & $\gamma_{2}^{(2)}$ \\
\hline 3 & 3.214 & & & 1 & $\mathrm{Zn}-\mathrm{O}$ & $\gamma_{3}^{(2)}$ \\
\hline 4 & 3.250 & & & 6 & $\mathrm{Zn}-\mathrm{Zn}$ & $\gamma_{4}^{(2)}$ \\
\hline 5 & 3.807 & & & 9 & $\mathrm{Zn}-\mathrm{O}$ & $\gamma_{5}^{(2)}$ \\
\hline \multicolumn{7}{|l|}{$g^{(3)}$} \\
\hline 1 & 1.977 & 1.977 & 108.11 & 6 & $\mathrm{O}-\mathrm{Zn}-\mathrm{Zn}$ & $\gamma_{1}^{(3)}$ \\
\hline 2 & 1.977 & 1.977 & 108.11 & 3 & $\mathrm{Zn}-\mathrm{O}-\mathrm{O}$ & $\gamma_{2}^{(3)}$ \\
\hline 3 & 1.977 & 1.977 & 110.80 & 6 & $\mathrm{O}-\mathrm{Zn}-\mathrm{Zn}$ & $\gamma_{3}^{(3)}$ \\
\hline
\end{tabular}

and $g_{3}^{(3)}$ peaks, respectively). A single effective multiplescattering signal $\eta^{(3)}$ including both the $\gamma^{(2)}$ and the $\gamma^{(3)}$ contributions will be used for the corresponding shells.

All XAFS signals $\gamma^{(n)}$ reported in Table I have been calculated up to $1000 \mathrm{eV}$ above the $\mathrm{Zn} K$-edge and considered for reproducing the experimental XAFS spectra collected under pressure.

In addition to the background, the $w$-XAFS calculated signal has been refined using a total number of 2 empirical parameters $\left(E_{0}\right.$, defining the alignment of the energy scale, and $S_{0}^{2}$, rescaling the calculated EXAFS signal) and 10 structural parameters: the six parameters describing the three two-body distributions (distance $R$ and corresponding variance $\sigma_{R}^{2}$ for each doublet) and the four parameters describing the two three-body distributions (angle mean value $\theta$ and corresponding variance $\sigma_{\theta}^{2}$ ). More sophisticated constraints are possible, however the sensitivity of further floating parameters (principally associated to the covariance matrix, $\sigma_{R \theta}^{2}, \sigma_{R_{1} R_{2}}^{2}$ for example) has been found to be almost zero. Therefore, the parameters of the covariance matrix associated to the three-atoms configuration have been confined in a narrow range of values around zero. The coordination numbers have been kept unchanged under pressure. The decomposition of the best-fit multiple-scattering signal into individual $\gamma^{(2)}$ and $\eta^{(3)}$ terms is shown in Figure 4. In all refinements, the value of the amplitude reduction factor $S_{0}^{2}$ has been found to lie around $0.95(5)$ and $E_{0}$ was found to coincide within $1 \mathrm{eV}$ with the energy position of the second peak of the experimental spectra derivative $(9663 \mathrm{eV})$.

\section{Rocksalt phase analysis}

All XAFS signals $\gamma^{(n)}$ reported in Table II have been calculated up to $1000 \mathrm{eV}$ above the $\mathrm{Zn} K$-edge and considered for reproducing the experimental XAFS spectra collected under pressure.



FIG. 4: Best-fit calculated multiple-scattering signals for the Zn $K$-edge XAFS $k \chi(k)$ spectra of $w$-ZnO recorded at ambient pressure. The $\gamma_{1}^{(2)}, \gamma_{3}^{(2)}$ and $\gamma_{5}^{(2)}$ signals are associated with the first, third and fifth shell of neighbors respectively. The two triplet signals are indicated as $\eta_{1}^{(3)}$ and $\eta_{3}^{(3)}$ because they include also the doublet associated with the longest interatomic distance of the triangle $\left(\gamma_{2}^{(2)}\right.$ and $\gamma_{4}^{(2)}$, respectively). Note the absence of the $\gamma_{2}^{(3)}$ signal because (1) its amplitude has been found to be too weak to be taken into account and (2) the longest distance of the corresponding atoms triplet does not involve the photoabsorbing atom ( $\mathrm{Zn})$. The agreement between measured (Expt) and calculated (Fit) spectra is excellent in the whole energy range.

The signal has been modeled as a sum of five contributions described as follow (the corresponding structural parameters are listed in brackets) : (1) First shell Zn$\mathrm{O} \gamma_{1}^{(2)}\left(R_{1}, \sigma_{R_{1}}^{2}\right) ;(2)$ three body O-Zn-Zn contribution $\eta_{1}^{(3)}$ associated with the $\theta_{1}=90^{\circ}$ triangles where the long bond coincides with peak 2 of the $g^{(2)}\left(R_{1}, \sigma_{R_{1}}^{2}, \theta_{1}\right.$, $\sigma_{\theta_{1}}^{2}$; the other covariance matrix parameters being fixed as for the $w$-ZnO XAFS analysis); (3) Third shell Zn$\mathrm{O} \gamma_{3}^{(2)}\left(R_{3}, \sigma_{R_{3}}^{2}\right)$; (4) three body O-Zn-Zn contribution $\eta_{1}^{(3)}$ associated with the $\theta_{1}=180^{\circ}$ triangles where the long bond coincides with peak 4 of the $g^{(2)}\left(R_{1}, \sigma_{R_{1}}^{2}, \theta_{1}\right.$, $\left.\sigma_{\theta_{1}}^{2}\right)$; (5) three body Zn-O-O contribution $\gamma_{2}^{(3)}$ associated with the $\theta_{1}=90^{\circ}$ triangles $\left(R_{1}, \theta_{1}, \sigma_{\theta_{1}}^{2}\right)$. The empirical parameters $E_{0}(\sim 9664 \mathrm{eV})$ and $S_{0}^{2}(\sim 0.90)$ have been fitted together with the structural parameters and the best-fit XAFS signals is shown in Fig 5. 
TABLE II: Pair and triplet configurations for $r$ - ZnO at ambient conditions. The three coordinates defining the three-body peaks are as described in Figure 3. The degeneracy (Deg) is specified for each configuration.

\begin{tabular}{|c|c|c|c|c|c|c|}
\hline Peak & $R_{12}(\AA)$ & $R_{13}(\AA)$ & $\theta_{213}\left(^{o}\right)$ & Deg. & Atoms & XAFS signal \\
\hline \multicolumn{7}{|l|}{$g^{(2)}$} \\
\hline 1 & 2.142 & & & 6 & $\mathrm{Zn}-\mathrm{O}$ & $\gamma_{1}^{(2)}$ \\
\hline 2 & 3.029 & & & 12 & $\mathrm{Zn}-\mathrm{Zn}$ & $\gamma_{2}^{(2)}$ \\
\hline 3 & 3.710 & & & 8 & $\mathrm{Zn}-\mathrm{O}$ & $\gamma_{3}^{(2)}$ \\
\hline 4 & 4.284 & & & 6 & $\mathrm{Zn}-\mathrm{Zn}$ & $\gamma_{4}^{(2)}$ \\
\hline \multicolumn{7}{|l|}{$g^{(3)}$} \\
\hline 1 & 2.142 & 2.142 & 90.0 & 24 & $\mathrm{O}-\mathrm{Zn}-\mathrm{Zn}$ & $\gamma_{1}^{(3)}$ \\
\hline 2 & 12.142 & 2.142 & 90.0 & 12 & $\mathrm{Zn}-\mathrm{O}-\mathrm{O}$ & $\gamma_{2}^{(3)}$ \\
\hline 3 & 2.142 & 2.142 & 180.0 & 6 & $\mathrm{O}-\mathrm{Zn}-\mathrm{Zn}$ & $\gamma_{3}^{(3)}$ \\
\hline
\end{tabular}



FIG. 5: Best-fit calculated multiple-scattering signals for the $\mathrm{Zn} K$-edge XAFS $k \chi(k)$ spectra of $r$-ZnO recorded at ambient pressure. Here again, the agreement between measured (Expt) and calculated (Fit) spectra is excellent.

\section{XANES}

From a qualitative point of view, the X-ray Absorption Near Edge spectroscopy is one of the most sensitive technique to probe pressure-induced structural modifications. Figure 6 shows raw Zn $K$-edge XANES data recorded as a function of pressure during the upstroke ramp, up to $\mathrm{P} \sim 16 \mathrm{GPa}$. In good agreement with EDX or EXAFS, the data shows the onset of a phase transition to the $\mathrm{NaCl}$ phase around $10 \mathrm{GPa}$, the transition being complete at about $14 \mathrm{GPa}$.

In principle, a complete recovery of the local geometry and site coordination around $\mathrm{Zn}$ could be obtained



FIG. 6: Zn $K$-edge XANES spectrum on bulk $\mathrm{ZnO}$ as a function of pressure during the upstroke ramp.

from the X-ray Absorption Near Edge Spectra. However, the quantitative analysis of this region presents difficulties mainly related to the theoretical approximation in the treatment of the potential and the need for heavy time-consuming algorithms to calculate the absorption cross section in the framework of a full multiple scattering approach. We have performed a comparison of the data with full multiple scattering calculations using a self-consistent energy dependent exchange correlation Hedin-Lundqvist potential (FEFF8 package [36]). Self consistency was obtained by successively calculating the electron density of states, electron density and Fermi level at each stage of the calculation within a 27 atoms cluster (4 $A$ or $\sim 3$ shells) centered on the atom for which the density of states is calculated, and then iterated. Full multiple scattering XANES calculations were carried out for a 93 atoms cluster ( $6.2 \AA$ or $\sim 7$ shells) centered on the absorbing atom: all multiple-scattering paths within this cluster were summed to infinite order. Besides the structural information defining the geometry of the cluster $(a=3.2427 \stackrel{\circ}{A}, c=5.1948 \stackrel{\circ}{A}$ and $u=0.3826$ for $w-Z n O$; $a=4.280 \AA$ for $r$-ZnO), the only external parameters used as input for the XANES simulations were a $0.5 \mathrm{eV}$ experimental broadening, and a small offset in the energy scale $(-5.0 \mathrm{eV})$. A typical result of the simulations for the wurtzite and $\mathrm{NaCl}$ phases of bulk $\mathrm{ZnO}$ are shown in Figure 7. The overall good agreement between the calculated and the measured spectra is striking, considering the absence of adjustable parameters in the theory (in particular, no structural disorder factor was added to the simulations). The major features and their variation 
TABLE III: 2- and 3-body distribution function peaks and associated interatomic distances as function of internal $(u)$ and unit-cell $(c, a)$ parameters of the wurtzite structure. The long-bond distance of the triplet peaks corresponds to the distance $R_{23}$ of Figure 3 .

\begin{tabular}{cccc}
\hline \hline Peaks & Atoms & Distances & Long-Bond Distances \\
$g_{1}^{(2)}$ & Zn-O & $u . c$ & \\
$g_{3}^{(2)}$ & Zn-O & $(1-u) \cdot c$ & \\
$g_{5}^{(2)}$ & Zn-O & $\sqrt{a^{2}+c^{2} \cdot u^{2}}$ & \\
$g_{2}^{(3)}$ & O-Zn-Zn & & $\sqrt{\frac{a^{2}}{3}+\frac{c^{2}}{4}}$ \\
$g_{3}^{(3)}$ & O-Zn-Zn & $a$ \\
\hline \hline
\end{tabular}

with pressure are correctly reproduced.

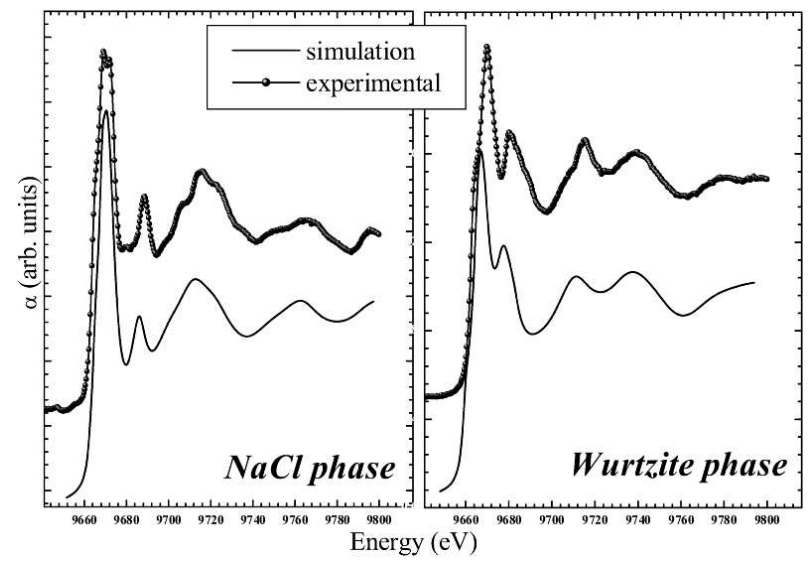

FIG. 7: Left: Zn $K$-edge XANES on bulk $r$-ZnO at $\mathrm{P}=15.6$ $\mathrm{GPa}$ and $a b$-initio simulated spectrum for the $\mathrm{NaCl}$ phase. Right: Zn $K$-edge XANES on bulk $w$-ZnO at $\mathrm{P}=0.4 \mathrm{GPa}$ and $a b$-initio simulated spectrum for the Wurtzite phase.

\section{RESULTS AND DISCUSSION}

\section{A. Wurtzite phase}

The structures of both $w$ - and $r$ - $\mathrm{ZnO}$ under pressure have been confirmed by comparing the XANES raw data with full multiple scattering calculations. In particular, the present study dispels the ambiguity on the crystallographic structure of the recovered sample at $\mathrm{P}=0$ which is found to be pure wurtzite, eliminating the previously proposed pure Rocksalt structure[10] or wurtzite-rocksalt mixture[12, 37].

Within the approximation described in Ref. [17], the EXAFS analysis was sufficiently sophisticated to determine both internal $(u)$ and unit-cell $(c, a)$ parameters as function of pressure. As a matter of fact, the wurtzite symmetry sets the relation between $u, c, a$ and the $g_{i}^{(n)}$ peaks position (see Table III).

The EXAFS results $a(P), c(P)$, and $V(P)$ are plotted in Figure 8 with both experimental X-ray diffraction and $a b$-initio results for comparison. Cell-parameter $(l(P))$ measurement as a function of pressure was analyzed with the Birch-Murnaghan equation of state: $l(P)=$ $l(0) \cdot\left[1+\frac{B^{\prime}}{B_{0}} \cdot P\right]^{\left(\frac{-1}{B^{\prime}}\right)}$, where $B^{\prime}$ is the pressure derivative of the bulk modulus and $B_{0}$ corresponds to the isothermal bulk modulus (or incompressibility) at $300 \mathrm{~K}$ (results summarized in Table IV).
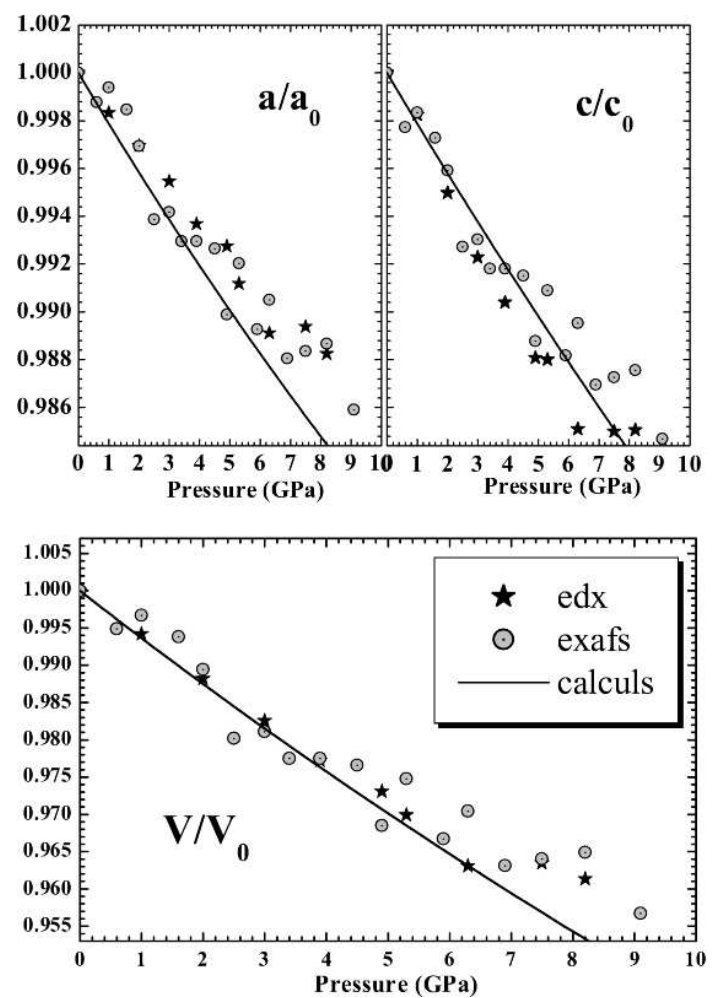

FIG. 8: Pressure dependence of the cell parameters for the wurtzite phase among compression.

The EXAFS results of $d_{Z n-O}(P)$ and $u(P)=$ $d_{Z n-O}(P) / c(P)$ are given in Figure 9 .

The variation of $d_{Z n-O}$ has been fitted with the BirchMurnaghan equation of state, leading to $B_{d 0}=420$ GPa (with $d_{Z n-O}(0)=1.985 \AA$ and $B_{d}^{\prime}$ fixed to 12 ). In good agreement with Desgreniers[11], the $u$ oxygen positional parameter indicates, within the experimental uncertainty, no peculiar change under pressure. Karzel et $a l[13]$ predicted an increase of $u$ to explain their ${ }^{67} \mathrm{Zn}$ Mössbauer spectroscopy results (pressure dependence of the main component $V_{z z}$ of the electric-field gradient tensor). However, this discrepancy with our result can stem from the fact that the $V_{z z}-u$ correlation has been indirectly deduced from LAPW calculations, $V_{z z}$ being measured at low temperature $(4.2 \mathrm{~K})$.

\section{B. Rocksalt phase}

In other II-VI compounds, the transition of the lowpressure to rocksalt high pressure phase is usually associ- 
TABLE IV: Birch-Murnaghan equation of state parameters for the wurtzite phase of ZnO. Superscripts "F" indicate a fixed value of $B^{\prime}$ in the Birch-Murnaghan fitting. Volumes, V in $\AA^{3}$, are per chemical formula unit. Distances $a$ and $c$ are in $\AA$. Isothermal bulk modulus are in GPa. $B_{i 0}$ defines the linear incompressibility along $i$ axis, $B_{i}^{\prime}$ its pressure derivative.

\begin{tabular}{cccccccccccc}
\hline \hline Refs & Method & $a_{0}$ & $B_{a 0}$ & $B_{a}^{\prime}$ & $c_{0}$ & $B_{c 0}$ & $B_{c}^{\prime}$ & $u_{0}$ & $V_{0}$ & $B_{0}$ & $B^{\prime}$ \\
\hline This work & EDX & 3.250 & 614 & $12^{F}$ & 5.201 & 406 & $12^{F}$ & - & 23.79 & 173 & $44^{F}$ \\
This work & EXAFS & 3.258 & 564 & $12^{F}$ & 5.220 & 561 & $12^{F}$ & 0.382 & 23.99 & 181 & $4^{F}$ \\
This work & DFT-LDA & 3.238 & 464 & 15 & 5.232 & 466 & 9 & 0.380 & 23.76 & 154 & 4.3 \\
{$[11]$} & X-ray & 3.2498 & - & - & 5.2066 & - & - & - & 23.810 & 142.6 & $3.6^{F}$ \\
{$[13]$} & X-ray+Mössbauer & 3.2496 & - & - & 5.2042 & - & - & - & 23.796 & 183 & $4^{F}$ \\
{$[14]$} & X-ray & 3.2475 & - & - & 5.2075 & - & - & - & 23.7847 & 136 & 9.4 \\
{$[37]$} & LDA & - & - & - & - & - & - & 0.379 & 22.874 & 162.3 & 4.05 \\
{$[37]$} & GGA & - & - & - & - & - & - & 0.3802 & 24.834 & 133.7 & 3.83 \\
{$[39]$} & Hartree-Fock & - & - & - & - & - & - & 0.3856 & 24.570 & 154.4 & 3.6 \\
{$[13]$} & LAPW & - & - & - & - & - & - & 0.381 & - & 160 & 4.4 \\
\hline \hline
\end{tabular}

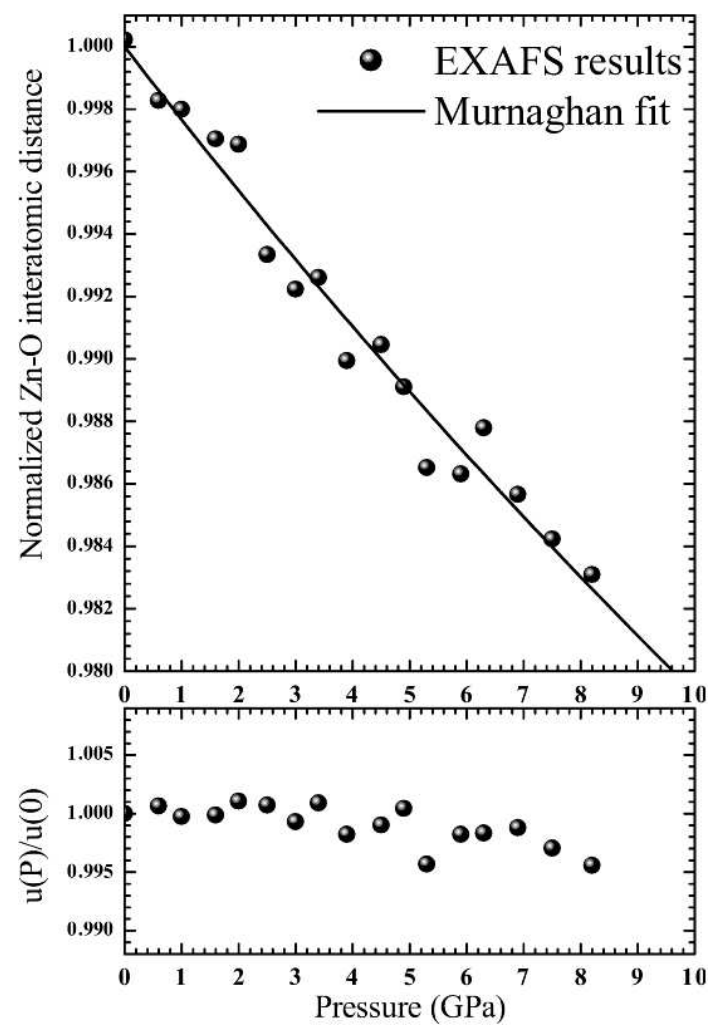

FIG. 9: Pressure dependence of the first nearest neighbors distance of zinc $\left(d_{Z n-O}\right)$ and the internal cell parameter $(u)$ for the wurtzite phase.

ated with a metallization. On the other hand, the present XANES analysis shows the $K$-edge energy position to increase at the $w$-to- $r$ transition, indicating an increase of the band gap. Further, no dramatic modification of the $r$ phase edge position up to $\sim 20 \mathrm{GPa}$ is observed, in good agreement with the absence of metallization previously determined through conductivity measurements [38].

The EXAFS and EDX results of $V(P)$ are given in Figure 10.

The pressure dependence of $d_{Z n-O}=\frac{a}{2}=\left(\frac{V}{2}\right)^{1 / 3}$

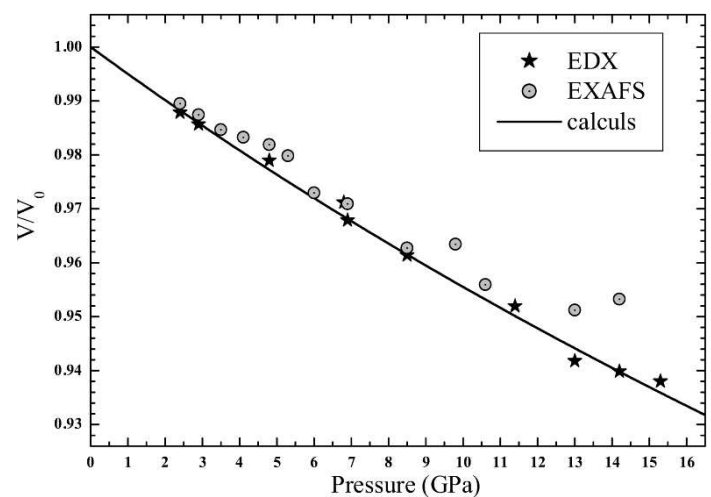

FIG. 10: Pressure dependence of the cell volume for the rocksalt phase upon decompression.

TABLE V: Birch-Murnaghan equation of state parameters for the rocksalt phase of $\mathrm{ZnO}$. Superscripts "F" indicate a fixed value of $B^{\prime}$ in the Birch-Murnaghan fitting. Volumes, $\mathrm{V}$ in $\AA^{3}$, are per chemical formula unit. Isothermal bulk modulus are in GPa.

\begin{tabular}{cccc}
\hline \hline Method & $V_{0}$ & $B_{0}$ & $B^{\prime}$ \\
\hline EDX & 19.64 & 204 & $4^{F}$ \\
EXAFS & 19.70 & 218 & $4^{F}$ \\
DFT-LDA & 19.49 & 198 & 4.6 \\
\hline
\end{tabular}

(where $a$ is the cubic cell parameter and $V$ the cell volume per chemical formula) has been fitted for decreasing pressure between 20 and 2 GPa with the Birch-Murnaghan equation of state. The results, given in Table $\mathrm{V}$, are in excellent agreement with previously reported data[10$14,37,39]$.

\section{Transition path}

From the $a b$-initio analysis (Fig 11), a kink in the pressure variation of $u$ is observed at $24 \mathrm{GPa}$, pressure slightly higher than the experimental $w$-to- $r$ pressure transition. 
The change of $u$ value from 0.382 (initial low pressure value) up to 0.5 is obviously connected to a symmetry change of the structure which can be seen as an intermediate phase $\left(w^{\prime}-\right)$ between $w$ - and $r$-ZnO [5]. As a matter of fact, this interpretation is of most interest, since in view of the $P-T$ phase diagram of $\mathrm{ZnO}$ [15], the $w$-to- $w^{\prime}$ transformation could be observable at low temperature, a domain where the pressure stability range of the $w$-phase is expected to be larger (against the rocksalt phase) than at $300 \mathrm{~K}$.

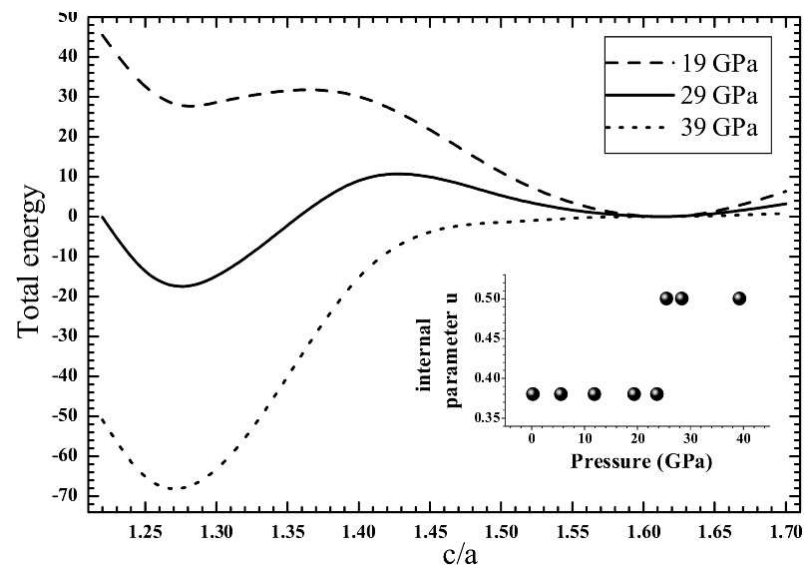

FIG. 11: Total energy of the wurtzite crystal with respect to the $\mathrm{c} / \mathrm{a}$ ratio. Inset: Calculated pressure dependence of the internal parameter $u$.

However, in opposition with previous conclusions [3, 5] predicting an homogeneous path along which $w$-ZnO is continuously transformed to $r$-ZnO, the present calculations lead to a constant value of $u$ below and above $24 \mathrm{GPa}$, pressure at which an abrupt discontinuity from 0.382 to 0.5 is observed. In the mean time, experimental data do not show instability of the wurtzite structure which could be expected below the $w$-to- $w^{\prime}$ transition. The previous EXAFS analysis gives a pressureindependent value of $u$ in the wurtzite stability range. From the XANES analysis, the structural phase transition is shown (Fig 12) to induce modifications in the electronic structure that lead to an energy shift of about $+1 \mathrm{eV}$ in the onset of absorption (defined as the energy corresponding to the maximum of the first derivative at the absorption edge). However, the $\mathrm{Zn} K$-edge energy for the wurtzite phase is unchanged under pressure. All these results lead to the conclusion that neither a pretransitional effect nor a second-order isostructural phase transition would be observed in the wurtzite phase prior to the first order transition to the rocksalt one.

Finally, it can be noticed that the elastic shear softening observed both experimentally[7] and theoretically[8] at room temperature (while $c / a$ is measured to be constant) is in disagreement with the transition path pro- posed in Ref [5]. Actually, the weakening of $C_{44}$ and $C_{66}$ elastic moduli under pressure is probably due to bondbending forces [40], in opposition with the proposed $w$ to- $w^{\prime}$ mechanism of transition where an initial decrease of $c / a$ ratio is expected before the opening of the bondbending angles. Thus, the existence of a $w$-to- $r$ transition path, as well as the nature of the intermediate phase, still remain an open question.

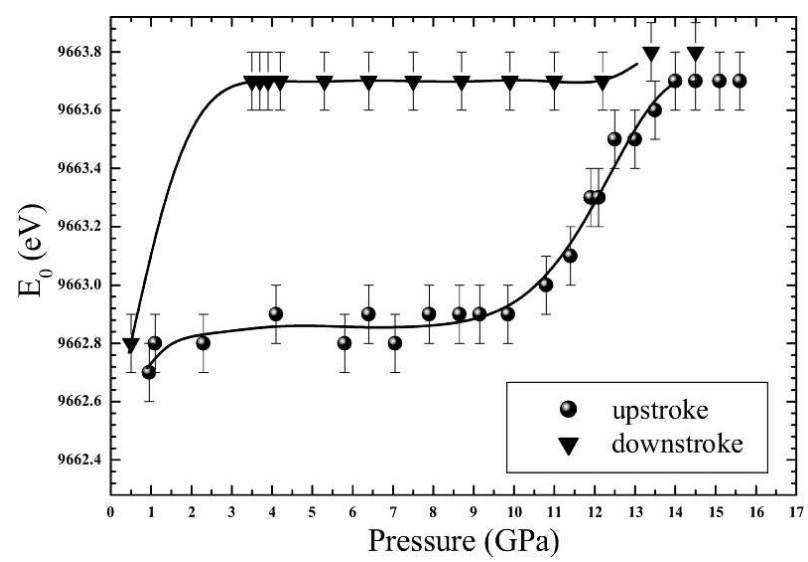

FIG. 12: Evolution of the $\mathrm{Zn} K$-edge absorption onset (defined as the energy value corresponding to the maximum of the first derivative at the absorption edge) with pressure for the pressure upstroke (circle) and downstroke (triangle) spectra respectively. The lines are a guide for the eyes.

\section{CONCLUSION}

Combined energy dispersive X-ray diffraction and Xray absorption spectroscopy experiments have been used to determined the internal parameter $u$ and the lattice parameters ratio $\frac{c}{a}$ of the wurtzite phase as function of pressure. In its experimental range of stability, the hexagonal wurtzite phase is shown to be undistorted by external hydrostatic pressures with no pre-transitional effect, in good agreement with present $a b$-initio calculations. The simulations have also been performed above the thermodynamical $w$-to- $r$ phase transition. While the expected shift of the $u$ value from 0.382 to 0.5 is observed, this transition is shown to be discontinuous in opposition with a possible displacive transformation.

\section{Acknowledgments}

The X-ray diffraction data has been analysis with DatLab (K. Syassen). We are grateful to Alberta Congeduti for her help during the experiments at LURE. We thank E. Principi for his useful comments on the manuscript. 
[1] S. Baroni, P. Giannozzi, and A. Testa, Phys. Rev. Lett. 58, 1861 (1987); P. Giannozzi, S. de Gironcoli, P. Pavone, and S. Baroni, Phys. Rev. B 43, 7231 (1991).

[2] V. Ozoliņš and A. Zunger, Phys. Rev. Lett. 82, 767 (1999).

[3] L. Bellaiche, K. Kunc and J. M. Besson, Phys. Rev. B 54, 8945 (1996).

[4] J. Serrano, A. Rubio, E. Hernández, A. Muñoz and A. Mujica, Phys. Rev. B 62, 16612 (2000).

[5] S. Limpijumnong and W.R.L. Lambrecht, Phys. Rev. Lett. 86, 91 (2001)

[6] Surprisingly, this prediction seems to be in disagreement with Serrano et al [4] who found a GaN wurtzite phase stable (with respect to the $w^{\prime}$ - structure) to about 200 GPa. To shed some light on that issue, we also performed the same type of calculation on $w$-GaN which appears to be stable versus $w^{\prime}$ - up to $500 \mathrm{GPa}$. However, Limpijumnong and Lambrecht do not give any quantitative data on the transition pressure, which could always be higher than $500 \mathrm{GPa}$.

[7] F. Decremps, J. Zhang, B. Li and R. C. Liebermann, Phys. Rev. B 63, 224105 (2001).

[8] A. M. Saitta and F. Decremps, in preparation.

[9] F. Decremps, J. Pellicer-Porres, A. M. Saitta, J.-C. Chervin and A. Polian, Phys. Rev. B 65, 092101 (2002).

[10] C.H. Bates, W.B. White and R. Roy, Science 137, 933 (1962).

[11] S. Desgreniers, Phys. Rev. B 58, 14102 (1998).

[12] J.M. Recio, M.A. Blanco, V. Luaña, R. Pandey, L. Gerward and J. Staun Olsen, Phys. Rev. B 58, 8949 (1998).

[13] H. Karzel, W. Potzel, M. Köferlein, W. Schiessl, U. Hiller, G.M. Kalvius, D.W. Mitchell, T.P. Das, P. Blaha, K. Schwartz and M.P. Pasternak, Phys. Rev. B 53, 11425 (1996).

[14] L. Gerward and J. Staun Olsen, J. Synchrotron Radiat. 2, 233 (1995).

[15] F. Decremps, J. Zhang and R. C. Liebermann, Eur. Phys. Lett. 51, 268 (2000).

[16] A. Filipponi, J. Phys.: Condens. Matter 7, 9343 (1995) and references therein.

[17] The measurement of $a(\mathrm{P}), c(\mathrm{P})$ through XAFS is in fact less straightforward. XAFS is a measurement of the correlated interatomic distance. Thus, due to the positive contribution of vibrations perpendicular to the bond $\left(\sigma_{\perp}^{2}\right)$, XAFS interatomic distances are expected to be slightly longer than XRD distances with a mismatch on the order of $\sigma_{\perp}^{2} / R$, decreasing with pressure (and increasing with temperature). It is not easy to correct the present results because the projection of vibration perpendicular to the bond direction is not known and should be measured independently. However, as a first approximation and within experimental errors, the vibrational effect can be neglected. A similar discussion could be find in A. Di Cicco, M. Taglienti, M. Minicucci and A. Filipponi, Phys. Rev. B 62, 12001 (2000) and references therein.

[18] R. Letoullec, J.P. Pinceaux and P. Loubeyre, High Pressure Res. 1, 77 (1988).

[19] G.J. Piermarini, S. Block, J.D. Barnett, and R.A. Forman, J. Appl. Phys. 46, 2774 (1975).

[20] H. K. Mao, J. Xu and B. M. Bell, J. Geophys. Res. B 91, 4673 (1986).

[21] H. Tolentino, E. Dartyge, A. Fontaine and G. Tourillon, J. Appl. Cryst. 21, 15 (1988).

[22] M. Hagelstein, A. San Miguel, A. Fontaine, and J. Goulon, J. Phys. IV France 7, C2 303 (1997).

[23] S. Pascarelli, O. Mathon and G. Aquilanti, J. Alloys and Compounds (2002).

[24] PWSCF code: www.pwscf.org.

[25] S. Baroni, P. Giannozzi, and A. Testa, Phys. Rev. Lett. 58, 1861 (1987); P. Giannozzi, S. de Gironcoli, P. Pavone, and S. Baroni, Phys. Rev. B 43, 7231 (1991).

[26] Troullier, N.; Martins, J.M., Phys. Rev. B 1991, 43, 1993.

[27] A. Filipponi and A. Di Cicco, Phys. Rev. B 52, 15135 (1995).

[28] A. Filipponi and A. Di Cicco, Phys. Rev. B 51, 12322 (1995).

[29] A. Filipponi and A. Di Cicco, Task quarterly 4 (4), 575 (2000) and references therein.

[30] A. Di Cicco, J. Phys. IV 7, 171 (1997).

[31] A. Mihelič, A. Kodre, I. Arčon, J. Padežnik Gomilšek and M. Borowski, Nucl. Instr. and Meth. in Phys. Res. B 196, 194 (2002).

[32] A. Di Cicco and F. Sperandini, Physica C 258, 349 (1996)

[33] J. Padežnik Gomilšek, A. Kodre, I. Arčon, A. M. LoireauLozac'h and S. Bénazeth, Phys. Rev. B 59, 3078 (1999).

[34] A. Di Cicco, M. De Crescenzi, R. Bernardini and G. Mancini, Phys. Rev. B 49, 2226 (1994); A. Filipponi, A. Di Cicco, P. Pianetta and T. Kendelwicz, Phys. Rev. B 53, 15571 (1996); V. Formoso, A. Filipponi, A. Di Cicco, G. Chiarello, R. Felici and A. Santaniello, Phys. Rev. B 61, 1871 (2000).

[35] A. Di Cicco, Phys. Rev. B 53, 6174 (1996).

[36] A. L. Ankudinov, B. Ravel, J.J. Rehr and S.D. Conradson, Phys. Rev. B 58, 7565 (1998).

[37] J. E. Jaffe, J. A. Snyder, Z. Lin, and A. C. Hess, Phys. Rev. B 62, 1660 (2000).

[38] J. Z. Jiang, J. S. Olsen, L. Gerward, D. Frost, D. Rubie and J. Peyronneau, Europhys. Lett., 50, 48 (2000).

[39] J. E. Jaffe and A. C. Hess, Phys. Rev. B 48, 7903 (1993).

[40] P.N. Keating, Phys. Rev. 145, 637 (1966). 